\title{
Atypical right hemisphere specialization for object representations in an adolescent with specific language impairment
}

\author{
Timothy T. Brown ${ }^{1,2,3}$ *, Matthew Erhart ${ }^{1,4}$, Daniel Avesar ${ }^{5}$, Anders M. Dale ${ }^{1,2,4}$, Eric Halgren ${ }^{1,2,4}$ and \\ Julia L. Evans ${ }^{6,7}$
}

${ }^{1}$ Multimodal Imaging Laboratory, University of California San Diego, La Jolla, CA, USA

${ }^{2}$ Department of Neurosciences, School of Medicine, University of California San Diego, La Jolla, CA, USA

${ }^{3}$ Center for Human Development, University of California San Diego, La Jolla, CA, USA

${ }^{4}$ Department of Radiology, School of Medicine, University of California San Diego, La Jolla, CA, USA

${ }^{5}$ Program in Experimental and Molecular Medicine, Dartmouth Medical School, Hanover, NH, USA

${ }^{6}$ Center for Research in Language, University of California San Diego, La Jolla, CA, USA

7 School of Behavioral and Brain Sciences, University of Texas Dallas, Dallas, TX, USA

\section{Edited by:}

Christos Papadelis, Harvard Medical

School, USA

\section{Reviewed by:}

Charline Urbain, The Hospital for Sick Children, Canada

Gerard Bastiaan Remijn, Kyushu

University, Japan

\section{${ }^{*}$ Correspondence}

Timothy T. Brown, Department of Neurosciences, UCSD School of Medicine, 8950 Villa La Jolla Drive, Suite C-101, La Jolla, CA 92037, USA e-mail: ttbrown@ucsd.edu
Individuals with a diagnosis of specific language impairment (SLI) show abnormal spoken language occurring alongside normal non-verbal abilities. Behaviorally, people with SLI exhibit diverse profiles of impairment involving phonological, grammatical, syntactic, and semantic aspects of language. In this study, we used a multimodal neuroimaging technique called anatomically constrained magnetoencephalography (aMEG) to measure the dynamic functional brain organization of an adolescent with SLI. Using single-subject statistical maps of cortical activity, we compared this patient to a sibling and to a cohort of typically developing subjects during the performance of tasks designed to evoke semantic representations of concrete objects. Localized patterns of brain activity within the language impaired patient showed marked differences from the typical functional organization, with significant engagement of right hemisphere heteromodal cortical regions generally homotopic to the left hemisphere areas that usually show the greatest activity for such tasks. Functional neuroanatomical differences were evident at early sensoriperceptual processing stages and continued through later cognitive stages, observed specifically at latencies typically associated with semantic encoding operations. Our findings show with real-time temporal specificity evidence for an atypical right hemisphere specialization for the representation of concrete entities, independent of verbal motor demands. More broadly, our results demonstrate the feasibility and potential utility of using aMEG to characterize individual patient differences in the dynamic functional organization of the brain.

Keywords: magnetoencephalography, specific language impairment, object concepts, semantic representations, hemispheric specialization, cerebral dominance

\section{INTRODUCTION}

Children with receptive or expressive language impairments who have normal hearing, an ordinary environment and rearing experiences, and show no other signs of developmental or neurological disorder are diagnosed with specific language impairment (SLI). Previously referred to as developmental aphasia or dysphasia, SLI is commonly encountered by speech-language clinicians and is found in disproportionate numbers of programs for children and adolescents with academic and behavioral dysfunction (Stark et al., 1988). The psycholinguistic manifestation of SLI can be highly variable across individuals, with primary impairments often evident within multiple aspects of language involving phonology, grammar, syntax, and semantics (Bishop, 2006).

Neurological and cognitive neuroscientific studies of functional brain organization demonstrate a prominent role of the left cerebral hemisphere generally in receptive and expressive language as well as specifically for the representation of semantic information, including word meanings and object concepts (Vigneau et al., 2006). A large body of research demonstrates that semantic knowledge about concrete entities is represented by distributed networks of discrete cortical regions most prominently involving large portions of the left temporal lobe and left ventral prefrontal cortex, as well as parietal and occipital areas (Martin and Chao, 2001; Binder and Desai, 2011), with these regions playing dissociable roles in relatively more perceptual versus conceptual processing. Despite undergoing significant developmental changes (Schlaggar et al., 2002; Brown et al., 2005; Szaflarski et al., 2006) and commonly involving regions of the right hemisphere as well (Martin and Chao, 2001; Binder and Desai, 2011; Donnelly et al., 2011), the typically developing cerebral functional organization for encoding word and object meanings shows a left hemisphere prominence (Martin, 1999) that is present even during infancy (Travis et al., 2011). 
Atypical hemispheric specialization in SLI has been suggested in the scientific literature since the early twentieth century (Orton, 1925), but evidence has been inconsistent, particularly within functional neuroimaging experiments (Whitehouse and Bishop, 2008). Volumetric postmortem and structural imaging studies generally have found right-greater-than-left asymmetries in temporal and inferior prefrontal regions in SLI (Jernigan et al., 1991; Plante et al., 1991; Gauger et al., 1997; De Fossé et al., 2004) and greater overall "right-heavy" asymmetry in higher-order association cortex in children with developmental language disorder as compared to the "left-heavy" profile typically shown by control children (Herbert et al., 2005). Pars triangularis (Broca's area) and perisylvian regions have been implicated specifically, found to be significantly smaller in SLI on the left or to show significantly greater rightward asymmetry (Gauger et al., 1997). At least one structural imaging study found no discernable differences between language impaired and typically developing children in unilateral measurements or bilateral left-right asymmetry of posterior intrasylvian anatomy (Preis et al., 1998).

Functional neuroimaging studies of language impairment have found evidence strongly suggestive of abnormal lateralization patterns in brain activity, but common methodological caveats have often limited a strong interpretation of the results. For example, Whitehouse and Bishop used functional transcranial Doppler ultrasonography (fTCD) to measure cerebral blood flow in 11 young adults with SLI during performance of a letter fluency task (Whitehouse and Bishop, 2008). Interestingly, they compared these subjects with young adults who had a childhood history of SLI but no longer met diagnostic criteria, as well as with adults with a diagnosis of autism and a control group. While silently generating words to a given letter, all of the participants in the SLI-history group and the majority of both the autistic and control subjects showed greater activation in the left compared to the right middle cerebral artery, interpreted by the authors as indicating left hemisphere dominance. In contrast, the majority of individuals with SLI showed brain activity that was deemed either strongly right-lateralized (54.5\%) or bilaterally prominent (27.3\%).

Atypical hemispheric specialization in SLI has also been suggested in the limited number of functional magnetic resonance imaging (fMRI) studies conducted to date (Hugdahl et al., 2004; Ellis Weismer et al., 2005; Dibbets et al., 2006; Badcock et al., 2012). For example, Badcock et al. compared structural and functional MRI measures during a language task in a group of eight individuals with SLI, their unaffected siblings, and typically developing controls. Anatomically, language impaired participants showed significantly more gray matter than controls in the left inferior frontal gyrus (IFG) and significantly less gray matter in bilateral superior temporal sulcus (STS) and in the right caudate nucleus. Physiologically, when activity during the performance of a covert naming task was contrasted with a silent baseline or passive listening to reversed speech, individuals with SLI showed reduced activity in comparison with the sibling and typical groups. Interestingly, these decrements in brain activity were localized to the same areas implicated in the structural morphological analysis. Furthermore, they observed "clearly left" lateralization of brain activity within the sibling and typical groups, but this was subjectively reduced in SLI. Brain-wide, there were no regions found that showed greater activation in SLI than the other groups. Had this been found, the authors state that it "might have been interpreted as evidence for different functional organization for language or compensatory or maladaptive reorganization." Badcock et al. also reported that patterns of brain activity in the SLI group were found to show more variability than the unaffected siblings and control group, as measured by laterality indices.

Despite the highly suggestive findings from these fTCD and fMRI studies, covert language tasks were used in these experiments, so no objective measures of subject task compliance and level of performance could be collected during scanning. Therefore, the greater heterogeneity in brain activity and overall under-activation by SLI could be explained simply by worse task performance within the clinical group (Murphy and Garavan, 2004), which would be expected for such tasks based on their diagnosis. Because of this, even for the sibling and control groups used for comparison, there is no way to be reasonably sure that the observed brain activity maps reflect physiological responses that were constrained to the cognitive processes of interest. This issue is common in developmental functional neuroimaging studies and limits the degree to which the desired conclusions can be drawn about the observed differences in functional brain organization (Brown et al., 2003, 2006; Palmer et al., 2004; Poldrack, 2010).

As many of the studies reviewed above point out, atypical cerebral dominance is not evident in all cases of poor language development, nor in all individuals with a diagnosis of SLI. Such patient heterogeneity in functional brain organization can contribute to equivocal results when comparisons are made between a clinical group and control group. Notably, group-averaged brain activity maps reveal only those functional neuroanatomical components that are most similar across subjects and will obscure individual differences. In groups that have especially high interindividual variability, averaged activity patterns may not be particularly representative of any individual. So, in order to achieve a clearer understanding of the relationships between cognitive functioning and functional brain organization, it may be useful to look more closely at individual patients, particularly with clinical groups such as SLI, which already have been shown to be cognitively and neurologically heterogeneous.

In addition, the majority of functional neuroimaging studies of SLI to date have used fMRI. Despite excellent spatial resolution, fMRI measures neural activity only indirectly, relying on a sluggish vascular response with poor temporal resolution. This inability to separate brain responses in time makes it considerably more difficult to isolate and identify specific cognitive functions that may be driving language task performance, such as sensory, perceptual, semantic, and motor processes (Posner, 1978, 2005; Cohen, 2011).

The purpose of the current study was to use a multimodal neuroimaging technique called anatomically constrained magnetoencephalography (aMEG) to localize with millisecond temporal sensitivity potentially atypical components of the functional brain organization within an individual patient. Here, we used a task paradigm designed specifically to engage cortical systems involved in the semantic representation of concrete objects in an individual with a diagnosis of SLI, comparing him to a group of typically developing individuals with no history of language problems. For comparison, we also applied identical methods to measure the 
dynamic functional brain organization of this patient's younger sister, who shows normal language abilities. Although differing by sex and age, she provides a useful comparison of the single-subject analysis methods.

For several reasons, we believed aMEG methods would provide a fruitful approach to the study of one patient. In addition to its sub-millisecond temporal resolution, aMEG provides excellent signal-to-noise properties and enhanced localization of brain activity through the use of noise-normalized source estimates constrained to the cortical reconstruction of each individual subject and aligned using sulcal and gyral surface-based registration (Dale et al., 2000; Dale and Halgren, 2001). Unlike single-dipole fitting MEG methods, the aMEG technique assumes multiple, distributed, and simultaneous cortical generators, which functional neuroimaging and recording methods overwhelmingly show is an appropriate assumption for cognitive tasks.

The primary questions posed in our experiment were: (1) does the dynamic functional brain organization for the semantic processing of concrete objects within an individual with a diagnosis and developmental history of SLI differ from that of a sample of typically developing individuals? (2) If so, how does the functional organization differ, topographically and temporally? (3) Specifically, does this individual show atypical aspects of the functional organization only during latencies that are associated with semantic encoding processes, or does he differ across all latencies measured? (4) Using the same conceptual and methodological approach, does the dynamic functional brain organization of an adolescent sibling with no history of language learning disorder mirror any of the differences found in the individual with SLI, or, instead, appear normal according to these methods? And more generally, (5) do aMEG techniques show feasibility and utility for mapping brain activity within individual patients, using activity distributions from a group of control subjects for direct comparison? In attempting to answer these questions, we hoped to assess both practical and substantive aspects of using aMEG to study individual differences in the dynamic functional organization of the brain.

\section{MATERIALS AND METHODS SUBJECTS}

One left-handed adolescent male diagnosed with specific language impairment (SLI-1; aged 17.8 years), 1 right-handed female sibling (Sib-1; aged 16.1 years), and a group of 12 typically developing right-handed individuals (mean age $=20.9$ years, $\mathrm{SD}=1.7$, range $=18.2-23.5$; five female) performed semantic processing tasks during MEG recording. The two individuals who were minors gave assent to participate with parental informed consent, and all control subjects gave informed consent using protocols approved by the UCSD Human Research Protections Program.

The primary characteristic of SLI is the failure to master spoken and written language expression and comprehension despite normal non-verbal intelligence, normal hearing acuity, and no overt physical causes, recognized syndromes, or mitigating medical factors known to cause language disorders in children. SLI-1 was diagnosed at age 4 years with expressive and receptive language delay, meeting criteria for SLI. He had no hearing or other sensory impairments and no history of serious medical problems. He has been followed clinically continuously since that time and received services during school age for language and auditory processing deficits - the only member of his family to qualify for such services. He was never diagnosed with a speech articulation disorder and so did not receive any speech-motor therapy, nor any other special services related to learning and development.

In late adolescence, SLI-1 continues to meet criteria for language impairment. Standard scores (population mean $=100$, $\mathrm{SD}=15)$ showed a squarely average non-verbal IQ (102, LeiterR; Roid and Miller, 1997) but below average performance on a language battery emphasizing grammar and semantics (82; Comprehensive Receptive and Expressive Vocabulary Test, Second Edition - CREVT-2; Wallace and Hammill, 2002). His receptive language score was 82 and expressive language score was 73 . Measures of comprehension of non-literal language, deriving meaning from context, and composite language knowledge ranged from about 2 to $2.5 \mathrm{SD}$ below average (72,60, and 62, respectively; Comprehensive Assessment of Spoken Language - CASL; Carrow-Woolfolk, 1999). On the Clinical Evaluation of Language Fundamentals (CELF-4; Semel et al., 2003), SLI-1 also scored within the clinical range on tests of formulating sentences and recalling sentences (five and six, respectively; mean/SD $=10 / 3$ ) but scored in the low average range on word classes (eight). On a measure of hand preference based on the Edinburgh questionnaire (Oldfield, 1971), SLI-1 reported being strongly left-handed.

On the same battery of measures, Sib-1 showed above-average non-verbal IQ (127) and above-average performance on a comprehensive language battery emphasizing semantics and grammar (120; CREVT-2). Her receptive language was 1 SD above average (115), and expressive language was within the average range (108). On standardized measures of the comprehension of non-literal meaning, deriving meaning from context, and composite language knowledge, Sib-1 scored within the average range (105, 93, and 99, respectively; CASL). On the CELF-4, she scored within the average to above-average range on tests of formulating sentences, recalling sentences, and word classes (13,11, and 14, respectively). She reported being strongly right-handed.

Typically developing control subjects were screened by interview and questionnaire to rule out history of developmental learning disorder, head injury, neurological or psychiatric disorder, or other major medical problems. Of the 12 control participants, 8 were undergraduate college or junior college students, and 4 were working full time. All were strongly right-handed. All denied currently taking psychotropic medication. All participants were screened for MRI and MEG safety by self-report and metal detector.

\section{MEG DATA ACOUISITION AND TASK PARADIGM}

Event-related fields were measured using a 306-channel wholehead Elekta NEUROMAG system inside a six-layer combination active-passive shielded room (IMEDCO-AG, Switzerland). The tasks, based on previously published studies of semantic processing (Dale et al., 2000; Marinkovic et al., 2003) were pilot-tested and modified to be more easily performed by children and adolescents and involved the presentation of two types of visual stimuli: printed words (high frequency, early acquired, highly imaginable concrete nouns; e.g., bed, mouse, door, whale, bug, 
house, leaf) and simple line drawings of lexically equivalent common, nameable objects. Picture and word stimuli were presented as white lines or letters against a black background. Subjects were instructed to respond to each item with laser-detected index finger lifts (one with the left hand, one with the right), indicating whether or not the object conveyed by word or image was small enough in size to fit into a shoebox. Stimuli were balanced with regard to the number of large versus small objects. Onscreen stimulus duration for words and pictures was $300 \mathrm{~ms}$, delivered with interstimulus intervals jittered between 3 and $5 \mathrm{~s}$. Words and pictures were presented roughly equal in size, subtending approximately $4^{\circ}$ of visual angle in their largest dimension. Picture and word stimuli were delivered in eight separate, alternating task runs (four picture runs, four word runs), each presenting 40 items and lasting about $4 \mathrm{~min}$.

\section{MRI DATA ACQUISITION}

High-resolution T1-weighted structural MRI scans optimized for gray/white matter contrast were acquired at $1.5 \mathrm{~T}$ for all subjects [time to echo $(\mathrm{TE})=3.8 \mathrm{~ms}$, time to repetition $(\mathrm{TR})=10.7 \mathrm{~ms}$, time to inversion $(\mathrm{TI})=1000 \mathrm{~ms}$, flip angle $=8^{\circ}$, trigger delay $(\mathrm{TD})=750 \mathrm{~ms}$, bandwidth $=31.25 \mathrm{~Hz} /$ pixel, field of view $($ FOV $)=24 \mathrm{~cm}$, matrix $=192 \times 192$, slice thickness $=1.2 \mathrm{~mm}]$. Real-time head motion tracking and correction was performed using PROMO, as described previously for prospective motion correction in spiral-navigated 3D pulse sequences (White et al., 2010). PROMO has been shown qualitatively and quantitatively to significantly improve image quality and reduce distortions caused by head motion and related artifacts, to increase the reliability of MR-derived brain measures (e.g., volume, thickness), and to improve the clinical diagnostic utility of structural MRI data when acquired in difficult-to-scan groups such as children (Brown et al., 2010; Kuperman et al., 2011).

\section{MULTIMODAL IMAGE PROCESSING AND ANALYSIS}

Multimodal brain activity maps were produced by generating a three-dimensional reconstruction of the cortical surface for each individual using MRI data (Fischl et al., 1998, 1999a,b; Dale et al., 1999) and spatially constraining the source estimations of MEGderived noise-normalized dipole strength to its geometry (Dale et al., 2000; Dale and Halgren, 2001). Dynamic statistical parametric maps (dSPMs) of cortical activity were computed for the two individuals and for the average of the typically developing group, spanning seven time windows based on previous aMEG studies of visual lexical semantic processing (Dale et al., 2000; Marinkovic et al., 2003; Leonard et al., 2010, 2011). Time windows were chosen to display separable brain activity events occurring across sensory, perceptual, and cognitive-semantic processing stages. At early latencies, beginning at $120 \mathrm{~ms}$, windows $50 \mathrm{~ms}$ wide were used to reveal brief, localized visual activity that has been observed in previous aMEG studies using visual semantic paradigms with similarly timed events (Dale et al., 2000; Marinkovic et al., 2003; Leonard et al., 2010, 2011). Rather than choosing single dSPM frames at several discrete latencies (that is, show maps restricted to exactly one selected time point, such as 120,158 , or $400 \mathrm{~ms}$ ), data were averaged within these time window blocks and displayed as such to provide a more complete representation of total brain activity over time, and one less prone to frame selection biases.
dSPMs were produced using only trials with correct task responses, reflecting cortical activity only during the successful evocation of semantic representations in every participant.

In order to more directly reveal how the dynamic patterns of cortical activity in SLI-1 and Sib-1 probabilistically compare with that of control subjects, and to avoid relying solely on qualitative evaluations of thresholded dSPM images, we also computed $z$-statistic maps showing the degree of similarity and dissimilarity in activity amplitude estimates at all cortical locations and all time points for both SLI-1 and Sib-1 in relation to the distribution of cortical activity for the comparison group, expressed in standard deviation (SD) units.

\section{RESULTS}

\section{BEHAVIOR}

During MEG recording, behavioral task accuracies were similar between the two siblings for both word (SLI-1=68\%; Sib$1=69 \%)$ and picture stimuli (SLI-1 = 83\%; Sib-1=80\%). Out of 160 total words, SLI-1 responded accurately to 109 items, and SIB-1 110. For pictures, SLI-1 responded correctly to 133 stimuli, and SIB-1 128 items. Response times (RTs) were somewhat slower for SLI-1 for both words [SLI-1 = $1044 \mathrm{~ms}$ (mean)/438 (SD); Sib$1=915 / 548]$ and pictures (SLI-1 = 877/269; Sib-1 = 766/302).

On average, typically developing subjects performed more accurately and faster than the siblings for both words $(89 / 6 \%$, range $=77-96 ; \mathrm{RT}=820 / 146$, range $=590-1063)$ and pictures $(87 / 7 \%$, range $=75-96 ; \mathrm{RT}=757 / 125$, range $=582-957)$. Out of 160 words total, the control group responded correctly on average to 142 items and ranged across individuals from 123 items correct to 154 . For picture stimuli, the mean number of items correct for the control group was 139 out of 160, with a range from 120 correct trials to 154 . Therefore, for word stimuli, both of the siblings performed with lower accuracy than the lowest performing control subject. For pictures, however, they both outperformed the lowest scoring control participant. As indicated by the RT ranges, both SLI-1 and Sib-1 responded, on average, faster for both words and pictures than the slowest typically developing control subject.

\section{IMAGING}

Noise-normalized dSPMs for the typically developing control group were strongly consistent with the results of previous aMEG and fMRI studies of the semantic processing of words and pictures (Dale et al., 2000; Dale and Halgren, 2001; Martin and Chao, 2001; Marinkovic et al., 2003; Vigneau et al., 2006; Leonard et al., 2010, 2011; Binder and Desai, 2011). During the processing of words, early lateral visual responses occurred between 120 and $170 \mathrm{~ms}$ in bilateral occipitotemporal regions and were stronger on the left (Figure 1). Within $50 \mathrm{~ms}$, activity spread across multiple regions bilaterally, including intraparietal and transverse occipital sulci, lateral occipitotemporal and temporal cortex, and anteriorly along perisylvian regions. By $300 \mathrm{~ms}$, cortical activity became more strongly left lateralized and included left frontal operculum after about $400 \mathrm{~ms}$. Qualitatively, Sib-1 showed a dynamic functional brain organization for processing words that was similar to the typically developing group. Her earliest lateral visual response occurred during $120-170 \mathrm{~ms}$ and was located in left 


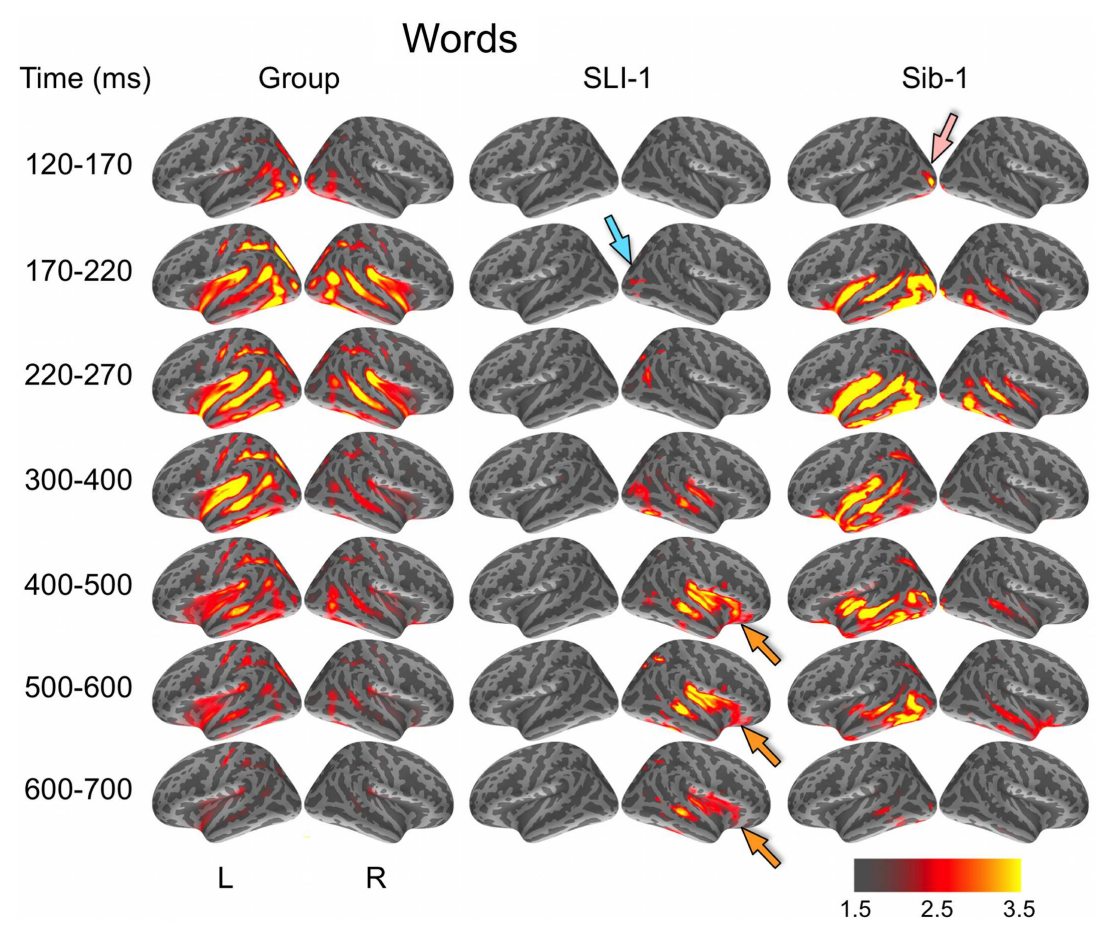

FIGURE 1 | Group and single-subject dSPMs of mean cortical activity during the semantic processing of words. In comparison to the functional organization of both the control group and sibling, SLI-1 showed strongly right-lateralized activity, from early sensoriperceptual to later cognitive stages. His early lateral occipital response was on the opposite side and somewhat delayed in time (blue arrow) in relation to his sister (pink arrow). During latencies typically associated with semantic encoding, he showed sustained activity within right temporal, perisylvian, and frontal opercular regions (orange arrows). Color scale represents square root of $F$ values, which are a measure of signal-to-noise. middle occipital sulcus (pink arrow). Activity then spread bilaterally and anteriorly along occipitotemporal and perisylvian regions and, similar to the group, became more strongly left lateralized at $300 \mathrm{~ms}$. Sustained left lateralized activity was apparent through $600 \mathrm{~ms}$ and at $500 \mathrm{~ms}$ included bilateral anterior insula and temporal poles.

In striking contrast to both the comparison group and to his younger sister, SLI-1 showed no cortical activity within the left hemisphere that surpassed the same threshold during the semantic processing of words. In general, his functional neuroanatomy was notable for being strongly right-lateralized, less distributed, and somewhat delayed in time. In contrast to the other subjects, the first discernable lateral visual response for SLI-1 occurred at 170$220 \mathrm{~ms}$ and was located within the right hemisphere (blue arrow). Activity then spread anteriorly more slowly and only on the right, engaging middle temporal, and right perisylvian regions only by about $300 \mathrm{~ms}$. From 400 to $700 \mathrm{~ms}$, SLI-1 showed sustained activity within right middle temporal, perisylvian, and frontal opercular areas (orange arrows).

During the evocation of semantic representations by picture stimuli, typically developing subjects showed spatiotemporal activity patterns that varied from word stimuli in ways consistent with previous aMEG studies. In general, neural activity was less strongly left lateralized, including early lateral visual responses within posterior occipitotemporal regions as well as later, from 300 to $600 \mathrm{~ms}$ (Figure 2). As with word processing, the dynamic functional organization shown by Sib-1 for pictures was similar to that of the comparison group, although qualitatively more strongly left lateralized. Her earliest lateral visual response was likewise apparent within the 120 - to 170 -ms time window, but only within middle and inferior occipital sulci on the left (pink arrow). Activity then spread anteriorly along left occipitotemporal and perisylvian regions and was weaker on the right than for the typically developing controls. From 300 to $500 \mathrm{~ms}$, activity for Sib-1 was somewhat more bilaterally evident for pictures than it was for words. Overall, her engagement of cortical areas during the presentation of pictures declined earlier than for words, especially within left anterior temporal and insular regions.

Just as for word stimuli, SLI-1 showed a functional neuroanatomy during the evocation of object concepts by pictures that were very different from both his sister and the comparison group. Again, his spatiotemporal patterns of activity were most notable for being strongly right-lateralized and somewhat delayed in time. Similar to words, picture stimuli evoked an early lateral visual response at 170-220 ms within right anterior occipital sulcus (blue arrow). Activity then spread first throughout proximal right occipital areas then involved right posterior perisylvian regions weakly. From 400 to 600 ms, SLI-1 showed sustained activity within right perisylvian and frontal opercular regions similar to (but weaker than) that observed during his semantic processing of words (orange arrows). 


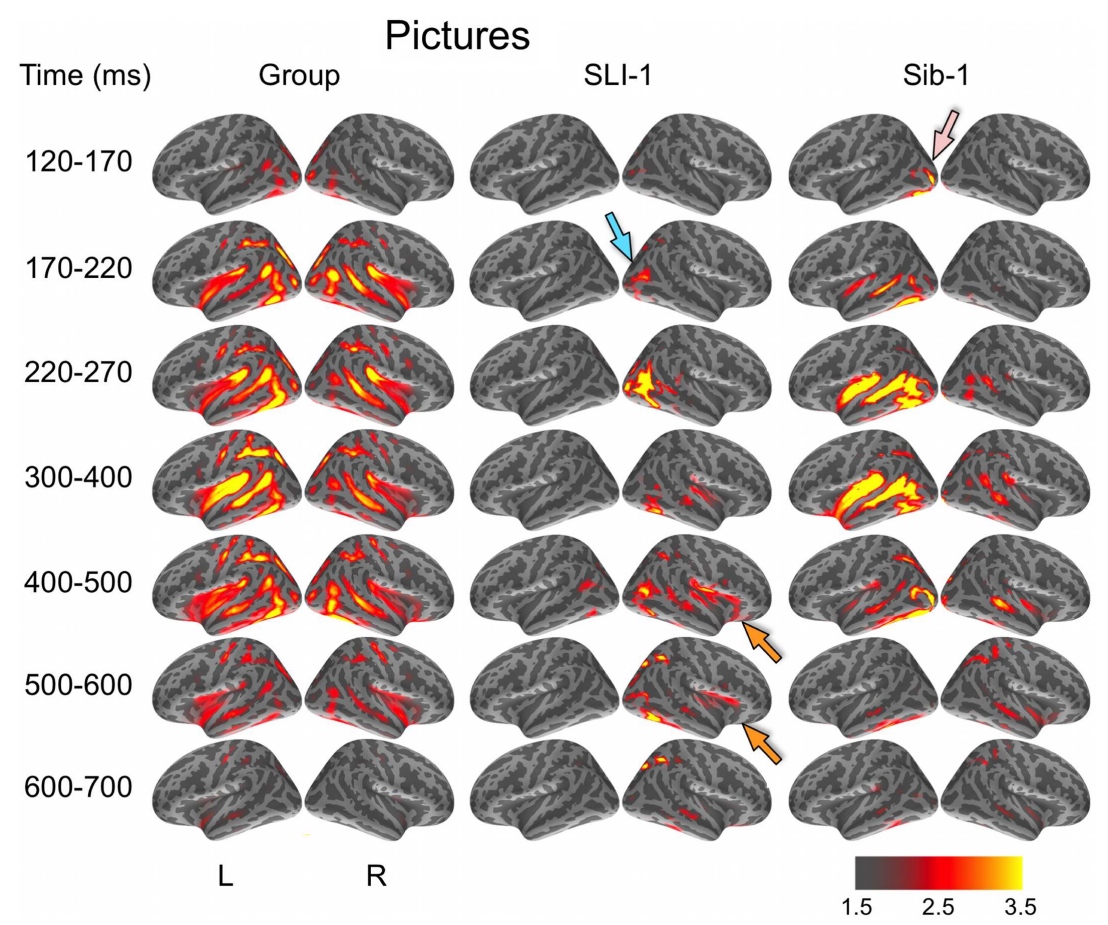

FIGURE 2 | Group and single-subject dSPMs of mean cortical activity during the semantic processing of pictures. In comparison to the functional organization of both the control group and sibling, SLI-1 showed strongly right-lateralized activity, from early sensoriperceptual to later cognitive stages. Similar to word processing, his early lateral occipital response was on the opposite side and somewhat delayed in time (blue arrow) in relation to his sister (pink arrow). During latencies typically associated with semantic encoding, he showed activity within right temporal, perisylvian, and frontal opercular regions (orange arrows), although weaker than for words. Color scale represents square root of $F$ values, which are a measure of signal-to-noise.
In a direct, vertex-wise comparison to the distribution of neural activity at all cortical locations and time points within the typically developing group using $z$-scores, SLI-1 showed differences from the typical dynamic functional organization that agreed with qualitative comparisons of the dSPMs. During the semantic processing of words, SLI-1 showed relative under-recruitment of many cortical regions bilaterally at early latencies, including perisylvian, anterior temporal, opercular, and lateral and superior frontal cortex (Figure 3). Beginning at $220 \mathrm{~ms}$, he showed the strongest areas of relative under-engagement within left frontal opercular and anterior temporal regions, continuing to $400 \mathrm{~ms}$. At the same time, he began to show notable relative over-recruitment of regions within the right occipital cortex (green arrow), which also extended to $400 \mathrm{~ms}$. At $400 \mathrm{~ms}$, SLI-1 showed greater activity than typically developing controls in several right hemisphere perisylvian cortical areas extending from subcentral sulcus to right frontal operculum. These regions showed sustained relative over-activity that continued from 500 to $700 \mathrm{~ms}$ (yellow arrows), where additional right hemisphere temporal and parietal overactivity also became apparent. When picture stimuli were used to evoke object representations, SLI-1 showed only relative underactivation within the left hemisphere and over-activation only within the right hemisphere. Just as for words, he showed early over-recruitment of right middle and inferior occipital regions beginning at $220 \mathrm{~ms}$ (green arrows). From 500 to $700 \mathrm{~ms}$, he showed late over-recruitment of right frontal regions similar to those for words (yellow arrows) and also over-recruitment of parietal, occipital, and temporal areas.

At the earliest latencies, the $z$-maps for Sib-1 revealed relative under-engagement of many bilateral anterior regions during word and picture processing that was similar to her brother. Interestingly, however, she showed notable relative over-recruitment of the left temporal pole in relation to the control group, which was consistently at 300-400 ms for both stimulus types (violet arrows). At late semantic processing stages, several cortical regions within right frontal and parietal, bilateral temporal, and left occipitotemporal cortex, showed greater levels of activity than the comparison group for pictures and words.

\section{DISCUSSION}

The primary purpose of our study was to test the feasibility and usefulness of anatomically constrained MEG for making direct comparisons between individual patients and typically developing control subjects in the dynamic functional organization of the brain. Using this multimodal functional neuroimaging technique, which provides the uncommon ability to localize cortical activity with millisecond temporal resolution, our findings revealed in an individual patient with a history of developmental language disorder an atypical right hemisphere specialization for the semantic representation of concrete entities. 

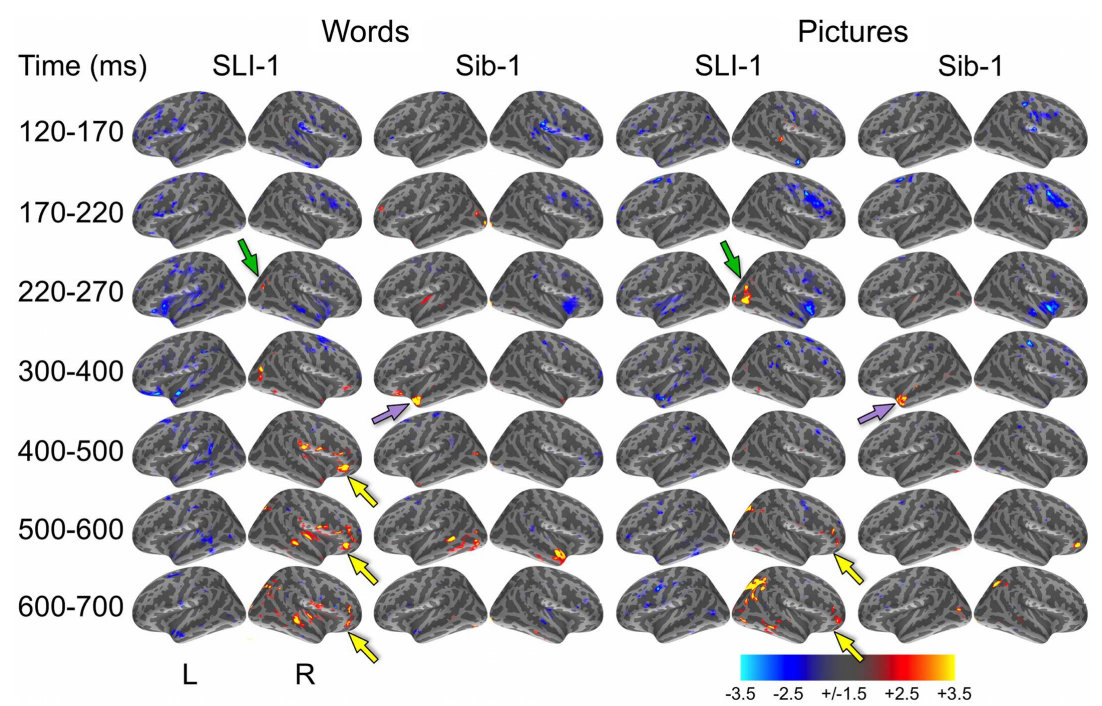

FIGURE 3 | z-Statistic maps of single-subject cortical activity in relation to the typically developing control group during the semantic processing of words and pictures. In direct comparison to the control group, SLI-1 showed relative under-activation of many left hemisphere regions and over-activation of only right hemisphere regions. This included early over-recruitment of right lateral occipital cortex for both words and pictures (green arrows), as well as later over-recruitment of right frontal opercular regions during semantic latencies (yellow arrows). Compared to controls, Sib-1 showed early under-engagement of right anterior regions for words and pictures, consistent over-engagement of left temporal pole 300-400 ms (violet arrows), and over-recruitment of several left and right areas at the latest time windows. Color scale represents $z$-statistics (standard deviation units).
Several aspects of our study support this interpretation. Using single-subject statistical maps, this patient showed strongly right-lateralized brain responses during the successful performance of a task that requires the evocation of semantic representations of visual objects when no spoken verbal response was required. Strong engagement of right hemisphere perisylvian regions was observed even during middle and late latencies typically associated with semantic encoding processes. His marked right hemisphere predominance was evident from early sensoriperceptual through later cognitive processing stages and was utilized for the semantic processing of both word and picture stimuli. During performance of the same tasks, typically developing control subjects, in contrast, showed bilateral involvement at early latencies followed by activity predominantly within the left hemisphere, especially during the processing of words. This is strongly consistent with the topography and timing from previous aMEG and fMRI studies of the semantic processing of pictures and words. As an additional control comparison for our single-subject analysis methods, the dynamic functional brain organization of a younger sibling with normal language development was found to be largely similar to the control group. Vertex-wise direct comparison of the patient and sibling to the distribution of cortical activity at every location and time point shown by the control group verified differences revealed by the comparison of independent brain activity maps.

Altogether, the functional neuroanatomical differences for this individual suggest a supramodal neural system for object concepts that appears similar to the left lateralized organization previously observed within association cortex in typically developing adults (Marinkovic et al., 2003), except that it is supported by the right hemisphere and appears to be engaged somewhat later in time. In bypassing reliance on auditory input, avoiding speech-motor demands, recording overt behavioral responses, mapping only successful trials, and localizing brain activity with millisecond temporal resolution, our interpretation of the functional organization can be more convincingly constrained to operations that involve semantic encoding and object representations. In contrast with traditional approaches testing cerebral functional specialization using naming, our findings were obtained without requiring speech-motor production components of language processing.

Language is made possible by a complex set of processing operations involving distributed mechanisms within the brain. Perhaps because of this, its development is surprisingly robust in the face of adverse neurological circumstances, such as early stroke, head trauma, and even hemispherectomy (Muller et al., 1998; Vicari et al., 2000; Bates et al., 2001; Fair et al., 2006; Liegeois et al., 2008; Trauner et al., 2013). This strongly suggests that there are multiple pathways to effective language learning and that the brain finds a detour when one pathway is blocked. Children who receive a clinical diagnosis of SLI, however, tend not to have a single problem cognitive area and instead display multiple underlying deficits (Bishop, 2006). By selectively probing object representations within only one patient, we hoped to learn something about the functional brain organization that might not be apparent from a group-averaged imaging study where patient heterogeneity in functional organization might produce equivocal results. Interestingly, we found strongly right-lateralized cortical responses within the very first individual with SLI we have tested.

Although these results strongly suggest an atypical functional brain organization for semantic processing, our study is limited 
in providing leverage to make inferences about several important etiological factors. Theories of abnormal right hemisphere involvement in developmental language disorders have existed for decades and emphasize atypical cerebral dominance for the motor control of speech and limb praxis (Zangwill, 1960; Satz, 1972; Geschwind and Galaburda, 1985). So, tasks used to test language lateralization typically employ an overt verbal production component such as spoken naming. However, even among left-handers such as SLI-1, who represent only about $10 \%$ of the world's population (Hardyck and Petrinovich, 1977), estimates of right hemisphere language dominance are thought to be relatively rare, ranging between about 7 and 27\% even when mixed/bilateral dominance is included (Rasmussen and Milner, 1977; Knecht et al., 2000; Drane et al., 2012). This means that only somewhere between 0.7 and $2.7 \%$ of the general population would be estimated to show right hemisphere or bilateral language dominance for spoken naming, the task commonly used in these studies. The fact that the large majority of left-handed individuals still seem to have left-dominant language representation suggests that it is common for dominant limb motor control to be decoupled from (i.e., contralateral to) dominant language representation.

The implications for the lateralization of the motor control of speech specifically, which is thought to follow lateralization for handedness, are unclear from available evidence. When language lateralization is probed using hemispheric anesthetization (i.e., the Wada procedure), are object concepts consistently represented within the hemisphere predominantly responsible for verbal motor control and speech production? Put another way, is a patient's inability to name objects in these experiments driven solely by arrest of the verbal articulators, by an inability to access the semantic representations required for naming, or by both? By disentangling these functions within an individual patient with a language learning disorder, we hoped to identify specifically whether object concepts themselves might be functionally organized in an atypical fashion, independent of verbal production demands. Perhaps some forms of developmental language learning disorder are caused by a mismatch between which hemisphere is dominant for speech-motor control and which specializes for the semantic encoding of object concepts and word meanings, causing access difficulties during language production. Although SLI-1 is strongly left-handed, this could be the case for his verbal praxis and will need to be tested further.

Our findings with SLI-1 are consistent with a number of cognitive developmental interpretations and models of hemispheric specialization, including possibly "weaker" semantic representations and the coarse encoding hypothesis (Beeman et al., 1994; Borovsky et al., 2013). His spontaneous task performance level, which provides one objective measure of the strength or accessibility of these semantic representations, might suggest that SLI-1's representations are no weaker than those of his sister, who performed similarly and nevertheless did not show strongly rightlateralized activity. Within the context of his clinical profile and developmental history, however, the present findings certainly suggest that his atypical functional brain organization is a contributor to and/or a product of his difficulties with language learning.

Interestingly, both SLI-1 and Sib-1 showed regions of sustained relative underactivity as compared to the control group during the processing of both words and pictures. This under-recruitment was most prominent at early latencies, from stimulus onset until about $270 \mathrm{~ms}$, and included perisylvian regions, particularly on the right for Sib-1. These effects may relate to speed of processing differences between these two individuals and the control group. Although both SLI-1 and Sib-1 responded, on average, faster for both words and pictures than the slowest typically developing control subject, their behavioral RTs were nevertheless slower than the average of the comparison group. SLI-1 responded on average $224 \mathrm{~ms}$ slower than the average of controls while processing words and $120 \mathrm{~ms}$ slower for pictures. Sib-1's average behavioral response was $95 \mathrm{~ms}$ slower than the control average for words but only $9 \mathrm{~ms}$ slower than that for control participants for pictures, suggesting that these early decrements in activity within the right hemisphere cannot be solely accounted for by slower task performance.

Several additional substantive issues and limitations with our study warrant further discussion. First, our group of control participants differed from one or both of the other individuals along two relevant characteristics: age and handedness. Because of this, we do not attempt to make any strong inferences about either the specific role of handedness in our findings or about the developmental state or phase of the individual subjects. Since this experiment employed a relatively untested collection of techniques as applied to single patients, we began by comparing SLI-1 to a group representative of typically developing adolescents and young adults comprised of right-handed individuals. Indeed, it would be interesting and informative to compare SLI-1 in the same way to an individual or group showing otherwise cognitively normal left-handedness. Such a comparison would be required to make inferences about the specific role of handedness in SLI-1's functional neuroanatomical differences. However, the scientific evidence would suggest, assuming a representative subject or sample of left-handed participants was obtained, that a direct comparison of SLI-1 with them might yield results similar to what we found in right-handers. Nevertheless, this is an empirical question that will require future experiments. The present study is only able to address the first-order question of how the dynamic functional brain organization for semantic processing in an individual with SLI differs from typically developing, right-handed controls.

Secondly, the range of ages for the control group was not ideal for making inferences about the two siblings in relation to agematched peers, since the control group was somewhat older. The individual with SLI was 3.1 years younger than the control group average and 0.4 years younger than the youngest control subject. His sister ( $\mathrm{Sib}-1$ ) was 4.8 years younger than the control group average and 2.1 years younger than the youngest control subject. So, we attempt to make no inferences about either of these individuals in relation to the ages or developmental phase of the control group. Instead, we have characterized brain activity in each participant both independently of the other participants and in direct relation to the distribution of brain activity from the same control group. So, their $z$-stat maps show levels of activity relative to the same distribution, making them a useful comparison with one another despite their age difference. Additionally, since SLI-1 is older than Sib-1, this comparison provides evidence that SLI1 's atypical functional organization cannot be solely attributed to 
being somewhat younger than the control sample. If this were true, his even younger sibling should show the same pattern.

That being said, available evidence from large-scale functional neuroimaging studies suggests that data from one 16- or 17-yearold participant would not be developmentally detectably different from that of individuals 18- to 23-years-old, because the developmental signal at these ages will be overpowered by the vast range of differences across individuals, even of the same age. From early school age into young adulthood, the range of individual differences variability in brain activity measures at a given age far exceeds the range of developmental changes that occur on average across even several years of development (Brown et al., 2005; Dosenbach et al., 2010). These studies, as well as positron emission tomography (PET) measurements of cerebral glucose metabolic rates (Chugani et al., 1987; Chugani and Phelps, 1991), also show that the slope of annualized developmental changes in activity decreases from late grade school age into adolescence and young adulthood, asymptoting during the ages studied here. This has been shown to be similar for many anatomical brain features as well, including morphological, diffusion, and signal intensity measures (Giedd et al., 1999; Sowell et al., 2003; Brown et al., 2012). Our data collected from Sib-1 demonstrate this point. Despite being about 2 years younger than the youngest control subject and about 5 years younger than the average age of the control group, the cortical regions that she engages during semantic processing show activity levels that fall predominantly within a similar dynamic range. Nevertheless, the specific ages of the control comparison group will become eminently more consequential when one seeks to make specific maturational or developmental inferences about the brain activity measures of one individual.

In light of our atypical findings for SLI-1, the data from the younger sibling become an especially useful comparison. Using methods identical to those applied with the language impaired adolescent, including use of the same statistical thresholds, both kinds of maps computed for Sib-1 (i.e., independent thresholded dSPMs and $z$-maps relative to the distributions of brain activity from the control group) revealed a functional brain organization that is largely similar to the control group. This provides evidence that the anomalous nature of the results found with SLI-1 cannot be explained simply by the single-subject analytic approach. The patterns of brain activity in a younger, language-typical individual - even from the same family - show a more typical functional organization for the same tasks.

Fortuitously, the siblings performed similarly on both types of cognitive tasks during MEG recording. This strengthens the confidence with which we can fairly compare their observed cortical functional organizations. Specifically, it further suggests that SLI-1's strongly rightward organization is not due solely to the fact that he was performing more poorly than the control group on average. If this were the case, Sib-1 would have shown similar right-lateralized activity. This point is important in light of recent MEG studies that have shown that right hemisphere participation in semantic decision tasks may increase with greater task difficulty in adults (Donnelly et al., 2011) or with objects that are never-before-seen and for which the names are newly encoded in children (Urbain et al., 2013). Interestingly, a very similar experimental paradigm using novel objects and names with adults showed instead that learning the names of new objects utilizes a cortical network very similar to the set of regions used for naming familiar items (Cornelissen et al., 2004). Important to note for all of these studies, left hemisphere activity was prominent despite relative increases in right hemisphere involvement. This progression from strong bilateral to reduced right hemisphere involvement during word learning is consistent with findings from studies of normal developmental changes in lexical semantic processing using both fMRI (Schlaggar et al., 2002; Brown et al., 2005; Szaflarski et al., 2006) and MEG (Ressel et al., 2008), as well as for second-language word learning in bilingual adults (Leonard et al., 2010, 2011).

More broadly, our results demonstrate the feasibility and potential utility of using aMEG to characterize individual differences in the cortical activity dynamics associated with specific cognitive functions. Scientifically and clinically, there are many reasons for developing improved functional neuroimaging methods to characterize single patients, not the least of which is to inform diagnostic assessment and individualized treatment planning. However, there have been technical, methodological, and conceptual barriers commonly encountered, such as weak signal-to-noise characteristics of the brain activity measures taken from only one patient, as well as the limited statistical approaches that can be adopted for making probabilistic comparisons and hypothesis tests based on data from a single-subject. Here, we used a whole-brain $z$-statistic technique that has been employed previously in case studies with fMRI data (Turkeltaub et al., 2004; Fair et al., 2006), which has the benefit of being conceptually straightforward but remains statistically descriptive.

Further development of multimodal functional neuroimaging approaches for single patients, such as with aMEG, will be crucial for providing a better understanding of the specific subcomponents underlying atypical brain-cognition-behavior linkages. Future aMEG experiments with both healthy and clinical subjects should focus on a more nuanced relation of the temporal dynamics of the functional brain organization to specific information processing operations, moving away from simple dichotomies involving broad psychological constructs such as left versus right "language dominance." Further, much work is needed in characterizing how this dynamic functional mosaic changes across different ages. We believe that more research into these kinds of distinctions will help refine our understanding of the role of hemispheric specialization in developmental language disorders and how sensoriperceptual processes, motor control, and semantic representations come together to support human language. This will undoubtedly aid in the early detection of developmental cognitive disorders, biologically inform our clinical diagnostic schemes, and improve our ability to individually tailor treatments.

\section{AUTHOR CONTRIBUTIONS}

Timothy T. Brown and Eric Halgren designed the experiment. Eric Halgren and Anders M. Dale developed the multimodal imaging methods. Timothy T. Brown, Matthew Erhart, and Daniel Avesar collected the MEG and MRI data, and Julia L. Evans collected and interpreted the behavioral data and performed the clinical diagnosis. Timothy T. Brown, Matthew Erhart, and Daniel Avesar 
processed and analyzed the imaging data. Timothy T. Brown wrote and edited the manuscript with input from the other authors.

\section{ACKNOWLEDGMENTS}

The authors gratefully thank the volunteer research subjects and parents who participated in this study. This study was funded in part by support from an Innovative Research Award from the Kavli Institute for Brain and Mind (Timothy T. Brown, Eric Halgren), by the National Institute of Neurological Disorders and Stroke (P50 NS022343; Timothy T. Brown, Eric Halgren, Anders M. Dale), and by the National Institute On Deafness and Other Communication Disorders (R01 DC005650; Julia L. Evans).

\section{REFERENCES}

Badcock, N. A., Bishop, D. V., Hardiman, M. J., Barry, J. G., and Watkins, K. E. (2012). Co-localisation of abnormal brain structure and function in specific language impairment. Brain Lang. 120, 310-320. doi:10.1016/j.bandl.2011.10.006

Bates, E., Reilly, J., Wulfeck, B., Dronkers, N., and Opie, M. (2001). Differential effects of unilateral lesions on language production in children and adults. Brain Lang. 79, 223-265. doi:10.1006/brln.2001.2482

Beeman, M., Friedman, R. B., Grafman, J., Perez, E., Diamond, S., and Lindsay, M. B. (1994). Summation priming and coarse semantic coding in the right hemisphere. J. Cogn. Neurosci. 6, 26-45. doi:10.1162/jocn.1994.6.1.26

Binder, J. R., and Desai, R. H. (2011). The neurobiology of semantic memory. Trends Cogn. Sci. (Regul. Ed.) 15, 527-536. doi:10.1016/j.tics.2011.10.001

Bishop, D. V. (2006). What causes specific language impairment in children? Curr. Dir. Psychol. Sci. 15, 217-221. doi:10.1111/j.1467-8721.2006.00439.x

Borovsky, A., Kutas, M., and Elman, J. L. (2013). Getting it right: word learning across the hemispheres. Neuropsychologia 51, 825-837. doi:10.1016/j. neuropsychologia.2013.01.027

Brown, T. T., Kuperman, J. M., Chung, Y., Erhart, M., McCabe, C., Hagler, D. J. Jr., et al. (2012). Neuroanatomical assessment of biological maturity. Curr. Biol. 22, 1-6. doi:10.1016/j.cub.2012.07.002

Brown, T. T., Kuperman, J. M., Erhart, M., White, N. S., Roddey, J. C., Shankaranarayanan, A., et al. (2010). Prospective motion correction of high-resolution magnetic resonance imaging data in children. Neuroimage 53, 139-145. doi:10. 1016/j.neuroimage.2010.06.017

Brown, T. T., Lugar, H. M., Coalson, R. S., Miezin, F. M., Petersen, S. E., and Schlaggar, B. L. (2005). Developmental changes in human cerebral functional organization for word generation. Cereb. Cortex 15, 275-290. doi:10.1093/cercor/bhh129

Brown, T. T., Petersen, S. E., and Schlaggar, B. L. (2003). Functional neuroimaging approaches to the study of human brain development. Perspect. Neurophysiol. Neurogenic Speech Lang. Disord. 13, 3-10. doi:10.1044/nnsld13.2.3

Brown, T. T., Petersen, S. E., and Schlaggar, B. L. (2006). Does human functional brain organization shift from diffuse to focal with development? Dev. Sci. 9, 9-11. doi:10.1111/j.1467-7687.2005.00455.x

Carrow-Woolfolk, E. (1999). Comprehensive Assessment of Spoken Language. Circle Pines, MN: American Guidance Services.

Chugani, H. T., and Phelps, M. E. (1991). Imaging human brain development with positron emission tomography. J. Nucl. Med. 32, 23-26.

Chugani, H. T., Phelps, M. E., and Mazziotta, J. C. (1987). Positron emission tomography study of human brain functional development. Ann. Neurol. 22, 487-497. doi:10.1002/ana.410220408

Cohen, M. X. (2011). It's about time. Front. Hum. Neurosci. 5:2. doi:10.3389/fnhum. 2011.00002

Cornelissen, K., Laine, M., Renvall, K., Saarinen, T., Martin, N., and Salmelin, R. (2004). Learning new names for new objects: cortical effects as measured by magnetoencephalography. Brain Lang. 89, 617-622. doi:10.1016/j.bandl.2003.12.007

Dale, A. M., Fischl, B., and Sereno, M. I. (1999). Cortical surface-based analysis. I. Segmentation and surface reconstruction. Neuroimage 9, 179-194. doi:10.1006/ nimg.1998.0395

Dale, A. M., and Halgren, E. (2001). Spatiotemporal mapping of brain activity by integration of multiple imaging modalities. Curr. Opin. Neurobiol. 11, 202-208. doi:10.1016/S0959-4388(00)00197-5

Dale, A. M., Liu, A. K., Fischl, B. R., Buckner, R. L., Belliveau, J. W., Lewine, J. D., et al. (2000). Dynamic statistical parametric mapping: combining fMRI and MEG for high-resolution imaging of cortical activity. Neuron 26, 55-67. doi:10.1016/S0896-6273(00)81138-1

De Fossé, L., Hodge, S. M., Makris, N., Kennedy, D. N., Caviness, V. S. Jr., McGrath, L., et al. (2004). Language-association cortex asymmetry in autism and specific language impairment. Ann. Neurol. 56, 757-766. doi:10.1002/ana.20275

Dibbets, P., Bakker, K., and Jolles, J. (2006). Functional MRI of task switching in children with specific language impairment (SLI). Neurocase 12, 71-79. doi:10.1080/13554790500507032

Donnelly, K. M., Allendorfer, J. B., and Szaflarski, J. P. (2011). Right hemispheric participation in semantic decision improves performance. Brain Res. 1419, 105-116. doi:10.1016/j.brainres.2011.08.065

Dosenbach, N. U., Nardos, B., Cohen, A. L., Fair, D. A., Power, J. D., Church, J. A., et al. (2010). Prediction of individual brain maturity using fMRI. Science 329, 1358-1361. doi:10.1126/science.1194144

Drane, D. L., Roraback-Carson, J., Hebb, A. O., Hersonskey, T., Lucas, T., Ojemann, G. A., et al. (2012). Cortical stimulation mapping and Wada results demonstrate a normal variant of right hemisphere language organization. Epilepsia 53, 1790-1798. doi:10.1111/j.1528-1167.2012.03573.x

Ellis Weismer, S., Plante, E., Jones, M., and Tomblin, J. B. (2005). A functional magnetic resonance imaging investigation of verbal working memory in adolescents with specific language impairment. J. Speech Lang. Hear. Res. 48, 405-425. doi:10.1044/1092-4388(2005/028)

Fair, D. A., Brown, T. T., Petersen, S. E., and Schlaggar, B. L. (2006). fMRI reveals novel functional neuroanatomy in a child with perinatal stroke. Neurology 67, 2246-2249. doi:10.1212/01.wnl.0000249348.84045.0e

Fischl, B., Dale, A. M., Sereno, M. I., Tootell, R. B., and Rosen, B. R. (1998). A coordinate system for the cortical surface. Neuroimage 7, S740.

Fischl, B., Sereno, M. I., and Dale, A. (1999a). Cortical surface-based analysis. II: inflation, flattening, and a surface-based coordinate system. Neuroimage 9, 195-207. doi:10.1006/nimg.1998.0396

Fischl, B., Sereno, M. I., Tootell, R. B., and Dale, A. M. (1999b). High-resolution intersubject averaging and a coordinate system for the cortical surface. Hum. Brain Mapp. 8, 272-284. doi:10.1002/(SICI)1097-0193(1999)8:4<272::AID-HBM10> 3.0.CO;2-4

Gauger, L. M., Lombardino, L. J., and Leonard, C. M. (1997). Brain morphology in children with specific language impairment. J. Speech Lang. Hear. Res. 40, 1272-1284.

Geschwind, N., and Galaburda, A. M. (1985). Cerebral lateralization. Biological mechanisms, associations, and pathology: III. A hypothesis and a program for research. Arch. Neurol. 42, 634-654. doi:10.1001/archneur.1985. 04060060019009

Giedd, J. N., Blumenthal, J., Jeffries, N. O., Castellanos, F. X., Liu, H., Zijdenbos, A., et al. (1999). Brain development during childhood and adolescence: a longitudinal MRI study. Nat. Neurosci. 2, 861-863. doi:10.1038/13158

Hardyck, C., and Petrinovich, L. F. (1977). Left-handedness. Psychol. Bull. 84, 385-404. doi:10.1037/0033-2909.84.3.385

Herbert, M. R., Ziegler, D. A., Deutsch, C. K., O’Brien, L. M., Kennedy, D. N., Filipek, P. A., et al. (2005). Brain asymmetries in autism and developmental language disorder: a nested whole-brain analysis. Brain 128, 213-226. doi:10.1093/brain/awh330

Hugdahl, K., Gundersen, H., Brekke, C., Thomsen, T., Rimol, L. M., Ersland, L., et al. (2004). FMRI brain activation in a Finnish family with specific language impairment compared with a normal control group. J. Speech Lang. Hear. Res. 47, 162-172. doi:10.1044/1092-4388(2004/014)

Jernigan, T. L., Hesselink, J. R., Sowell, E., and Tallal, P. A. (1991). Cerebral structure on magnetic resonance imaging in language- and learning-impaired children. Arch. Neurol. 48, 539-545. doi:10.1001/archneur.1991.00530170103028

Knecht, S., Drager, B., Deppe, M., Bobe, L., Lohmann, H., Floel, A., et al. (2000). Handedness and hemispheric language dominance in healthy humans. Brain 123, 2512-2518. doi:10.1093/brain/123.12.2512

Kuperman, J. M., Brown, T. T., Ahmadi, M. E., Erhart, M. J., White, N. S., Roddey, J. C., et al. (2011). Prospective motion correction improves diagnostic utility of pediatric MRI scans. Pediatr. Radiol. 41, 1578-1582. doi:10.1007/s00247-0112205- 1

Leonard, M. K., Brown, T. T., Travis, K. E., Gharapetian, L., Hagler, D. J. Jr., Dale, A. M., et al. (2010). Spatiotemporal dynamics of bilingual word processing. Neuroimage 49, 3286-3294. doi:10.1016/j.neuroimage.2009.12.009

Leonard, M. K., Torres, C., Travis, K. E., Brown, T. T., Hagler, D. J. Jr., Dale, A. M., et al. (2011). Language proficiency modulates the recruitment of non-classical 
language areas in bilinguals. PLoS ONE 6:e18240. doi:10.1371/journal.pone. 0018240

Liegeois, F., Connelly, A., Baldeweg, T., and Vargha-Khadem, F. (2008). Speaking with a single cerebral hemisphere: fMRI language organization after hemispherectomy in childhood. Brain Lang. 106, 195-203. doi:10.1016/j.bandl.2008.01.010

Marinkovic, K., Dhond, R. P., Dale, A. M., Glessner, M., Carr, V., and Halgren, E. (2003). Spatiotemporal dynamics of modality-specific and supramodal word processing. Neuron 38, 487-497. doi:10.1016/S0896-6273(03)00197-1

Martin, A. (1999). Automatic activation of the medial temporal lobe during encoding: lateralized influences of meaning and novelty. Hippocampus 9, 62-70. doi:10.1002/(SICI)1098-1063(1999)9:1<62::AID-HIPO7>3.0.CO;2-K

Martin, A., and Chao, L. L. (2001). Semantic memory and the brain: structure and processes. Curr. Opin. Neurobiol. 11, 194-201. doi:10.1016/S0959-4388(00) 00196-3

Muller, R. A., Chugani, H. T., Muzik, O., and Mangner, T. J. (1998). Brain organization of motor and language functions following hemispherectomy: a [(15)O]-water positron emission tomography study. J. Child Neurol. 13, 16-22. doi: $10.1177 / 088307389801300103$

Murphy, K., and Garavan, H. (2004). Artifactual fMRI group and condition differences driven by performance confounds. Neuroimage 21, 219-228. doi:10.1016/ j.neuroimage.2003.09.016

Oldfield, R. C. (1971). The assessment and analysis of handedness: the Edinburgh inventory. Neuropsychologia 9, 97-113. doi:10.1016/0028-3932(71)90067-4

Orton, S. T. (1925). "Word blindness" in school children. Arch. Neurol. Psychiatry 14, 581-615. doi:10.1001/archneurpsyc.1925.02200170002001

Palmer, E. D., Brown, T. T., Petersen, S. E., and Schlaggar, B. L. (2004). Investigation of the functional neuroanatomy of single word reading and its development. Sci. Stud. Read. 8, 203-223. doi:10.1207/s1532799xssr0803_2

Plante, E., Swisher, L., Vance, R., and Rapcsak, S. (1991). MRI findings in boys with specific language impairment. Brain Lang. 41, 52-66. doi:10.1016/0093934X(91)90111-D

Poldrack, R. A. (2010). Interpreting developmental changes in neuroimaging signals. Hum. Brain Mapp. 31, 872-878. doi:10.1002/hbm.21039

Posner, M. I. (1978). Chronometric Explorations of Mind. Hillsdale, NJ: Erlbaum.

Posner, M. I. (2005). Timing the brain: mental chronometry as a tool in neuroscience. PLoS Biol. 3:e51. doi:10.1371/journal.pbio.0030051

Preis, S., Jancke, L., Schittler, P., Huang, Y., and Steinmetz, H. (1998). Normal intrasylvian anatomical asymmetry in children with developmental language disorder. Neuropsychologia 36, 849-855. doi:10.1016/S0028-3932(98)00033-5

Rasmussen, T., and Milner, B. (1977). The role of early left-brain injury in determining lateralization of cerebral speech functions. Ann. N. Y. Acad. Sci. 299, 355-369. doi:10.1111/j.1749-6632.1977.tb41921.x

Ressel, V., Wilke, M., Lidzba, K., Lutzenberger, W., and Krageloh-Mann, I. (2008). Increases in language lateralization in normal children as observed using magnetoencephalography. Brain Lang. 106, 167-176. doi:10.1016/j.bandl.2008.01.004

Roid, G. H., and Miller, L. J. (1997). Leiter International Performance Scale-Revised. Wood Dale, IL: Stoelting Co.

Satz, P. (1972). Pathological left-handedness: an explanatory model. Cortex 8, 121-135. doi:10.1016/S0010-9452(72)80013-3

Schlaggar, B. L., Brown, T. T., Lugar, H. M., Visscher, K. M., Miezin, F. M., and Petersen, S. E. (2002). Functional neuroanatomical differences between adults and school-age children in the processing of single words. Science 296, 1476-1479. doi:10.1126/science.1069464

Semel, E., Wiig, E. H., and Secord, W. A. (2003). Clinical Evaluation of Language Fundamentals, 4th Edn. San Antonio, TX: The Psychological Corporation.

Sowell, E. R., Peterson, B. S., Thompson, P. M., Welcome, S. E., Henkenius, A. L., and Toga, A. W. (2003). Mapping cortical change across the human life span. Nat. Neurosci. 6, 309-315. doi:10.1038/nn1008
Stark, R. E., Tallal, P., and McCauley, R. J. (1988). Language, Speech and Reading Disorders in Children: Neuropsychological Studies. Austin, TX: Pro-Ed.

Szaflarski, J. P., Schmithorst, V. J., Altaye, M., Byars, A. W., Ret, J., Plante, E., et al. (2006). A longitudinal functional magnetic resonance imaging study of language development in children 5 to 11 years old. Ann. Neurol. 59, 796-807. doi:10.1002/ana.20817

Trauner, D. A., Eshagh, K., Ballantyne, A. O., and Bates, E. (2013). Early language development after peri-natal stroke. Brain Lang. 127, 399-403. doi:10.1016/j. bandl.2013.04.006

Travis, K. E., Leonard, M. K., Brown, T. T., Hagler, D. J. Jr., Curran, M., Dale, A. M., et al. (2011). Spatiotemporal neural dynamics of word understanding in 12- to 18-month-old-infants. Cereb. Cortex 21, 1832-1839. doi:10.1093/cercor/bhq259

Turkeltaub, P. E., Flowers, D. L., Verbalis, A., Miranda, M., Gareau, L., and Eden, G. F. (2004). The neural basis of hyperlexic reading. An FMRI case study. Neuron 41, 11-25. doi:10.1016/S0896-6273(03)00803-1

Urbain, C., Bourguignon, M., Op de Beeck, M., Schmitz, R., Galer, S., Wens, V., et al. (2013). MEG correlates of learning novel objects properties in children. PLoS ONE 8:e69696. doi:10.1371/journal.pone.0069696

Vicari, S., Albertoni, A., Chilosi, A. M., Cipriani, P., Cioni, G., and Bates, E. (2000). Plasticity and reorganization during language development in children with early brain injury. Cortex 36, 31-46. doi:10.1016/S0010-9452(08)70834-7

Vigneau, M., Beaucousin, V., Herve, P. Y., Duffau, H., Crivello, F., Houde, O., et al. (2006). Meta-analyzing left hemisphere language areas: phonology, semantics, and sentence processing. Neuroimage 30, 1414-1432. doi:10.1016/j.neuroimage. 2005.11.002

Wallace, G., and Hammill, D. D. (2002). Comprehensive Receptive and Expressive Vocabulary Test, 2nd Edn. Austin, TX: Pro-Ed.

White, N., Roddey, C., Shankaranarayanan, A., Han, E., Rettmann, D., Santos, J., et al. (2010). PROMO: real-time prospective motion correction in MRI using image-based tracking. Magn. Reson. Med. 63, 91-105. doi:10.1002/mrm. 22176

Whitehouse, A. J., and Bishop, D. V. (2008). Cerebral dominance for language function in adults with specific language impairment or autism. Brain 131, 3193-3200. doi:10.1093/brain/awn266

Zangwill, O. L. (1960). Cerebral Dominance and Its Relation to Psychological Function. Springfield, IL: C. C. Thomas.

Conflict of Interest Statement: Anders M. Dale and Eric Halgren are founders of and hold equity interest in Cortechs Labs, La Jolla, CA, USA and serve on its scientific advisory board. The terms of this arrangement have been reviewed and approved by UCSD in accordance with its conflict of interest policies. The other co-authors declare that the research was conducted in the absence of any commercial or financial relationships that could be construed as a potential conflict of interest.

Received: 02 October 2013; accepted: 03 February 2014; published online: 14 February 2014.

Citation: Brown TT, Erhart M, Avesar D, Dale AM, Halgren E and Evans JL (2014) Atypical right hemisphere specialization for object representations in an adolescent with specific language impairment. Front. Hum. Neurosci. 8:82. doi: 10.3389/fnhum.2014.00082

This article was submitted to the journal Frontiers in Human Neuroscience.

Copyright (c) 2014 Brown, Erhart, Avesar, Dale, Halgren and Evans. This is an openaccess article distributed under the terms of the Creative Commons Attribution License (CC BY). The use, distribution or reproduction in other forums is permitted, provided the original author(s) or licensor are credited and that the original publication in this journal is cited, in accordance with accepted academic practice. No use, distribution or reproduction is permitted which does not comply with these terms. 